\title{
Supporting Information for: "Comparing an all-atom and a coarse-grained description of lipid bilayers in terms of enthalpies and entropies: from MD simulations to 2D lattice models"
}

\author{
Davit Hakobyan and Andreas Heuer \\ Institute of Physical Chemistry, WWU Muenster, Corrensstr. 28/30, 48149 Muenster, Germany \\ Center for Multiscale Theory and Computation (CMTC), WWU Muenster, 48149 Muenster, Germany
}

\section{Note 1}

The distributions of the order parameter for reference AA (330 K and $340 \mathrm{~K}$ ) and CG systems ( $290 \mathrm{~K}$ and $300 \mathrm{~K}$ ) are shown in Figure S1. The figure also contains the distributions of the same AA systems where the tail carbon atoms are grouped together to form one bead coordinate as a result of averaging the coordinates of the 4 atoms. This is denoted CG-type order parameter and denoted in Figure S1 as 4in1. This mapping is performed to compare the distributions of the AA system and the CG system with definitions of order parameters, based on the same number of degrees of freedom. The mapped AA system shows a similar distribution of order parameters as the CG system.

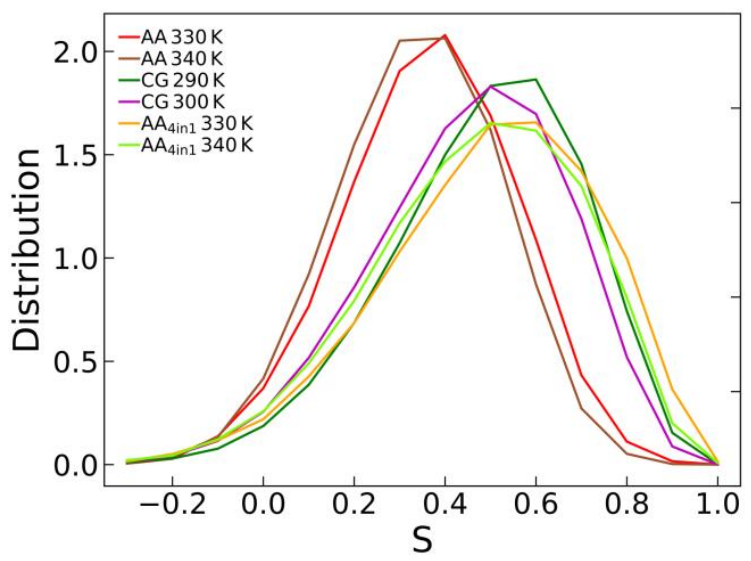

Figure S1. The order parameter distributions at $330 \mathrm{~K}$ and $340 \mathrm{~K}$ for the AA DPPC system (with the standard definition of the order parameter as well as with the CG-type order parameter as defined in the text) and at $290 \mathrm{~K}$ and $300 \mathrm{~K}$ for the CG system. 


\section{Note 2}

The polynomials of pairwise interaction energies and self-interaction energies are specified in Table S1 and Table S2, respectively.

Table S1.Pairwise interaction energy polynomial coefficients as a function of average order parameter within the $S$ range from -0.3 to 1.0 , starting with term of $0^{\text {th }}$ order.

\begin{tabular}{rr}
\hline DPPC - DPPC (AA) & DPPC - DPPC (CG) \\
\hline-66.192 & -35.290 \\
14.296 & 2.921 \\
-17.170 & 3.496 \\
12.547 & -26.092 \\
-115.641 & 5.220 \\
84.159 &
\end{tabular}

Table S2. Self-interaction energy polynomial coefficients as a function of order parameter within the $\mathrm{S}$ range from -0.3 to 1.0 , starting with the term of $0^{\text {th }}$ order.

\begin{tabular}{rr}
\hline DPPC - DPPC (AA) & DPPC - DPPC (CG) \\
-1.200 & -0.072 \\
10.013 & -0.295 \\
-8.320 & -10.485 \\
-39.644 & 9.190 \\
-115.281 & -9.068 \\
238.514 & \\
-114.475 & \\
\hline
\end{tabular}

The nearest neighbor (NN) functions for all-atom CHARMM36 (AA) and MARTINI coarsegrained (CG) systems are two variable polynomials (depend on temperature and order parameter) and are of rather complex shape. For simplicity the Python code is presented in Table S3 and Table S4 for calculating the nearest neighbor functions for AA and CG cases, respectively. 


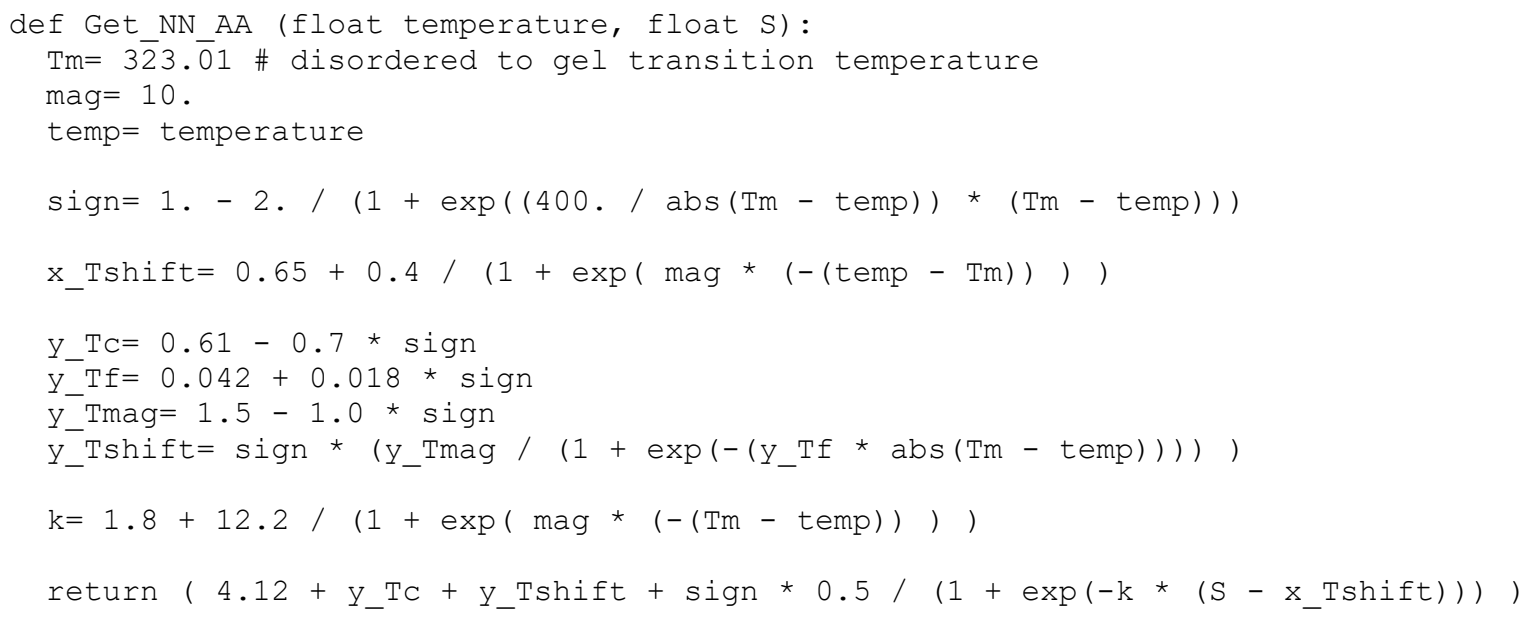

Table S4. NN function for MARTINI coarse-grained DPPC lipid

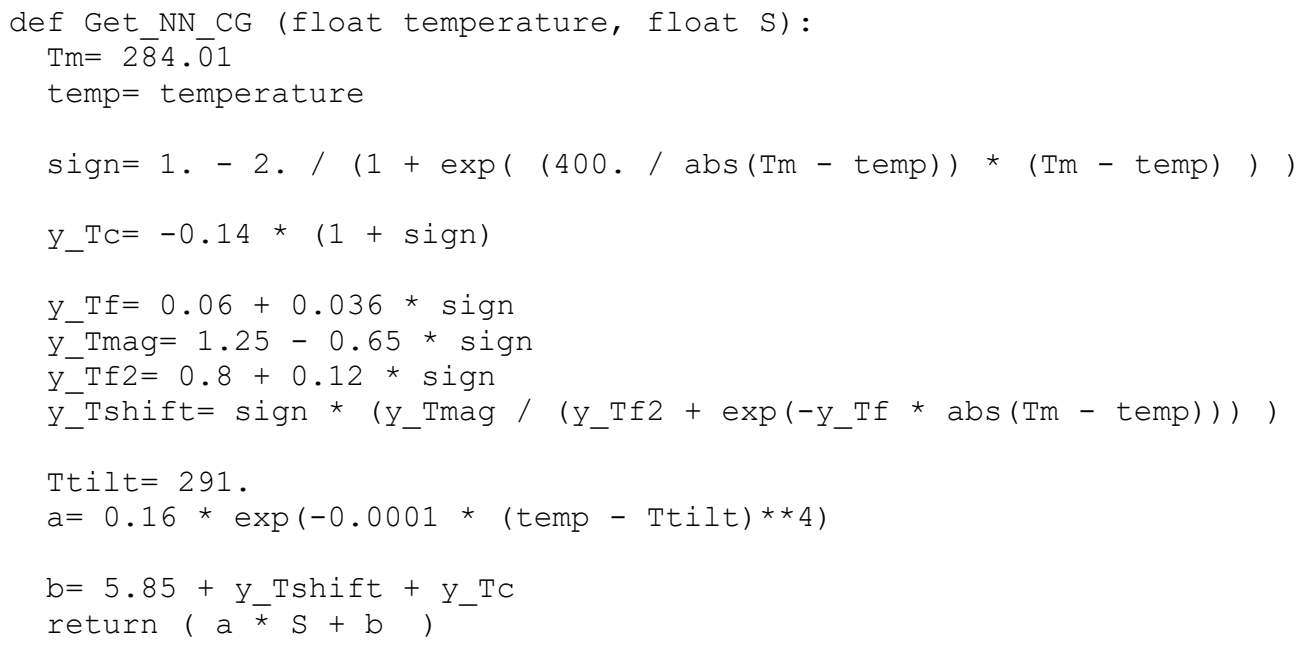

The resulting NN curves are shown in Figure S2 for different temperatures for AA and CG cases to reproduce the MD NN data. 

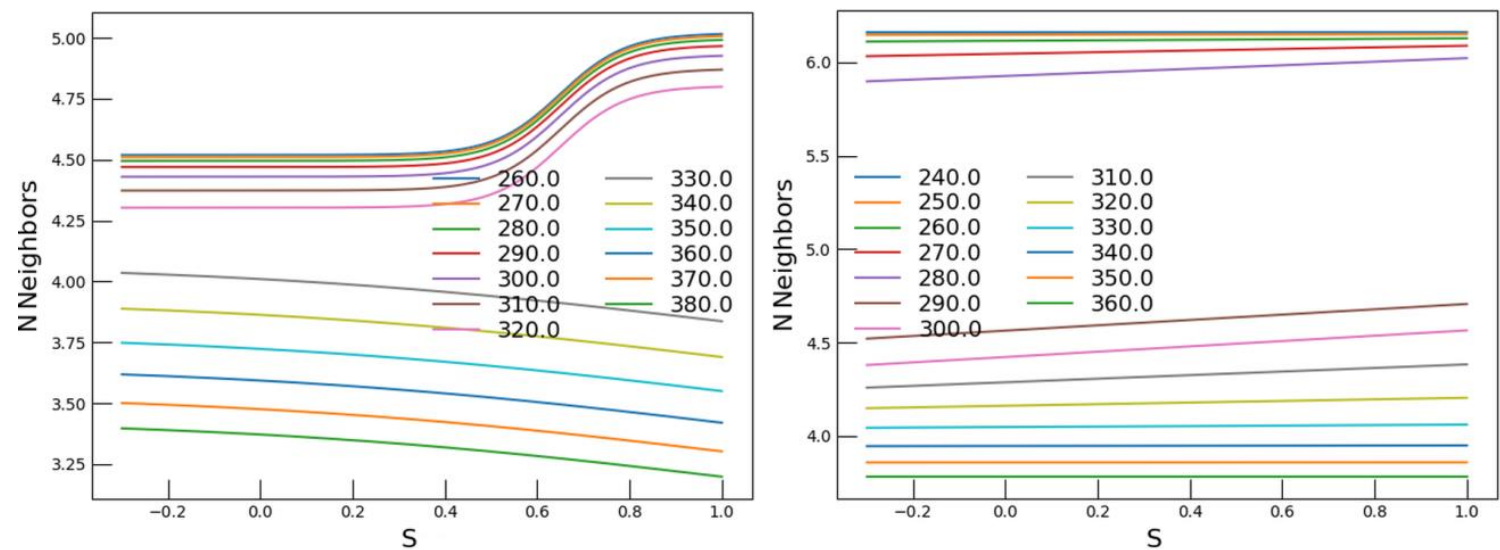

Figure S2. The nearest neighbor polynomial as a function of order parameter S and temperature for all-atom CHARMM36 (left) and MARTINI coarse-grained (right) pure DPPC systems.

The polynomials of configurational entropies are shown in Table S5 for temperatures $330 \mathrm{~K}$ and $290 \mathrm{~K}$ for AA and CG force fields, respectively.

Table S5. Entropic polynomial coefficients, starting with the term of $0^{\text {th }}$ order. These polynomial functions demonstrate the best fitting to rather complex entropic curves.

\begin{tabular}{rr}
\hline DPPC (AA) & DPPC (CG) \\
-6.28 & -4.48 \\
19.86 & 7.80 \\
-26.24 & -19.38 \\
3.61 & -10.10 \\
-100.16 & 86.11 \\
-169.90 & -236.31 \\
1540.75 & 249.97 \\
-4245.44 & -99.32 \\
4844.18 & \\
16003.88 & \\
-90005.15 & \\
191276.03 & \\
-212295.37 & \\
121381.35 & \\
-28257.26 & \\
\hline
\end{tabular}




\section{Note 3}

For comparison the entropy terms obtained, using temperature-independent $\mathrm{NN}$ function (originally proposed) and temperature-dependent NN functions, are shown in Figure S3 for the AA DPPC systems. Both entropies are calculated using the $\mathrm{S}$ distribution at a temperature of 330 $\mathrm{K}$.

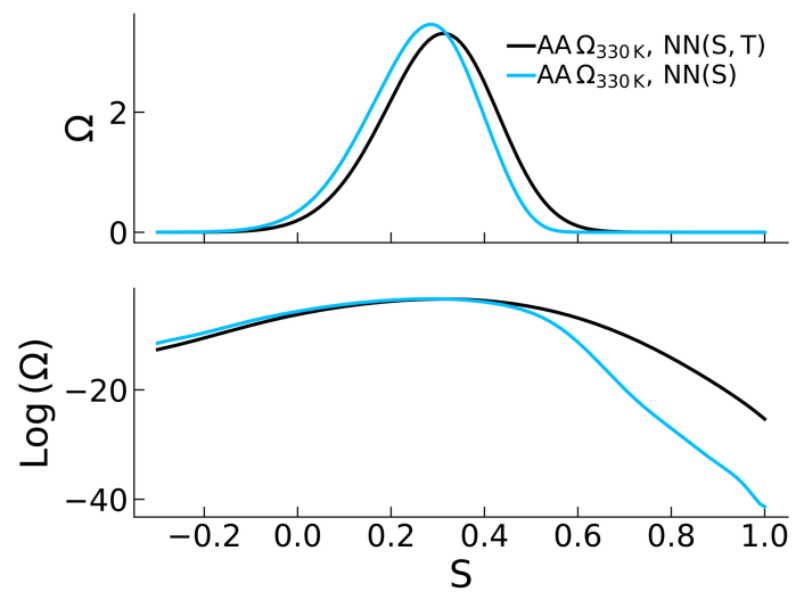

Figure S3. The AA DPPC new (black) and original (blue) entropy curves obtained using temperature-dependent and temperature-independent nearest neighbor functions. Both curves are calculated using the $\mathrm{S}$ distribution at $330 \mathrm{~K}$. 


\section{Note 4}

During the gelation process long-living defects in AA and CG MD systems can be evaluated e.g. by determining the formation and persistence of clusters of lipids with low order parameter values. Such cluster persistence is shown in Figure S4 where the low S clusters are tracked over some predefined periods.

Here, two lipids are considered to be in a cluster if they are neighbors and their order parameters fit into the slot of \pm 0.2 around a binned $\mathrm{S}$ value. In the above plot the vertical $\mathrm{S}$ axis is binned by a step of size 0.4 (starting from -0.3 up to 1.0) and the clusters at each slot are shown by their own colors. At order parameter values lower than 0 no cluster is found. The minimum cluster size considered here is assumed to be formed by at least three lipids. Each circle in the plot represents a cluster. The circle size is proportional to the cluster size found at a given time, while the center of the circle is the average order parameter of the lipids in the cluster. The connecting lines between circles show the existence of intersecting lipids between clusters at time $t_{1}$ and the next time $t_{2}$. The thickness of the lines is proportional to the ratio $(25 \%, 50 \%, 75 \%$ and $100 \%)$ of the number of intersecting lipids and the number of lipids found in the cluster at time $t_{1}$.

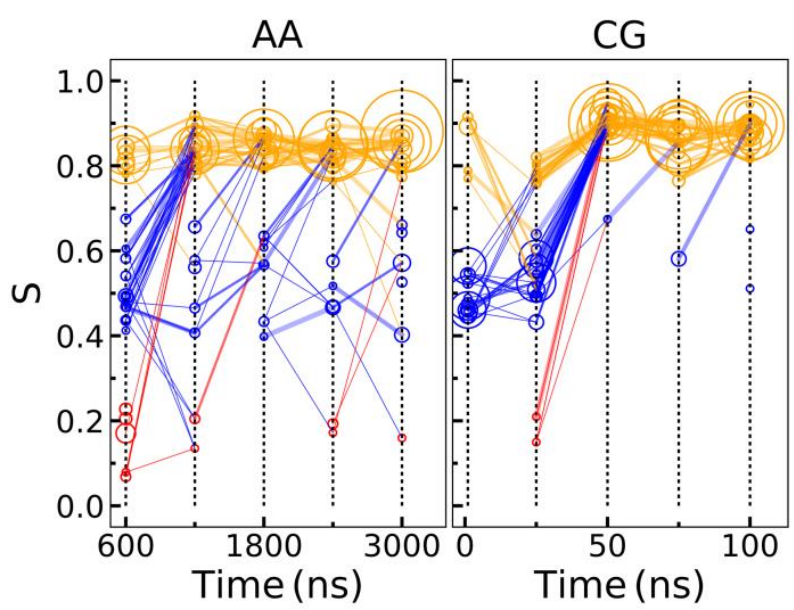

Figure S4. The persistence of low order parameter clusters in AA DPPC system at temperature $320 \mathrm{~K}$ (left) and CG DPPC system at temperature $280 \mathrm{~K}$ (right). The clusters differ by three colors corresponding to three different order parameter ranges. A cluster has to contain at least three lipids. The circles represent clusters were the radius is proportional to the cluster size (the smallest circle contains only three lipids). The connecting lines indicate the presence of common lipids in clusters defined at time $t_{1}$ and the next time $t_{2}$ (dashed vertical lines). The thickness of the lines is proportional to the ratio $(25 \%, 50 \%, 75 \%$ and $100 \%)$ of the number of intersecting lipids and the number of lipids found in the cluster at time $t_{1}$.

The figure suggests that there is only a minor number of small defects in the AA system. The thin lines between the circles (representing clusters) indicate that only part of the lipids (usually not more than 50\%) keep their disorder after a period of $600 \mathrm{~ns}$. Such clusters become fewer with time, i.e. after $1800 \mathrm{~ns}$ just a few of them remain. Thus, the main source of disorder comes from 
stochastic changes of single-lipid or pair of lipids domains. For CG systems at $280 \mathrm{~K}$ the defects seem to exist only for the first $150 \mathrm{~ns}$ after which the system is, basically, found in a defect-free gel state. 